\title{
IDEMPOTENTS IN GROUP ALGEBRAS
}

\author{
BY WALTER RUDIN ${ }^{1}$ \\ Communicated November 26, 1962
}

I. Introduction. If $G$ is a group, its group algebra $L^{1}(G)$ consists of all complex functions $f$ on $G$ for which the norm

$$
\|f\|=\sum_{x \in G}|f(x)|
$$

is finite; addition is pointwise, and multiplication is defined by convolution:

$$
(f * g)(x)=\sum_{y \in G} f(y) g\left(y^{-1} x\right) .
$$

Any $f \in L^{1}(G)$ for which

$$
f * f=
$$

will be called an idempotent on $G$.

The support of a complex function $f$ on $G$ is the set of all $x \in G$ at which $f(x) \neq 0$. The support group of $f$ is the smallest subgroup of $G$ which contains the support of $f$.

By methods involving Fourier transforms and the Pontryagin duality theory, the idempotents on abelian groups are completely known [2, p. 199]. (For nondiscrete locally compact abelian groups, the classification of the idempotent measures was completed by P. J. Cohen [1].) Let us draw attention to the following facts, of which (A) and (D) are probably the most striking:

(A) If $f$ is an idempotent on an abelian group $G$, then the support group of $f$ is finite.

(B) Idempotents on abelian groups are self-adjoint (i.e., $f\left(x^{-1}\right)$ is the complex conjugate of $f(x)$ ).

(C) On a finite abelian group there are only finitely many idempotents (namely $2^{n}$ if the group has $n$ elements). On a countable abelian group there are at most countably many idempotents.

(D) If $f$ is an idempotent on an abelian group and if $\|f\|>1$, then $\|f\| \geqq \frac{1}{2} \sqrt{ } 5[3$, p. 72$]$. (Note that there are no idempotents $f$ with $\|f\|<1$, except $f=0$.)

It is the purpose of the present note to show that each of the above statements becomes false if the word "abelian" is omitted.

'Sponsored by the Mathematics Research Center, United States Army, Madison, Wisconsin under Contract No. DA-11-022-ORD-2059, and by NSF grant GP-249. 
II. Consider a set $E$ which contains the integers and the three symbols $\alpha, \beta, \gamma$, let

$$
\begin{aligned}
& a=(\alpha \beta \gamma), \\
& b=(\beta \gamma)\left(\cdots-2-1 \quad \begin{array}{llllll}
\cdots & \cdots & 1 & \cdots
\end{array}\right)
\end{aligned}
$$

be permutations of $E$, and let $G$ be the group generated by $a$ and $b$. The relations

$$
a^{3}=1, \quad b^{2 k-1} a=a^{2} b^{2 k-1}
$$

hold for all integers $k$, and $G$ consists of the distinct elements

$$
a^{n} b^{k} \quad(n=0,1,2 ; k=0, \pm 1, \pm 2, \cdots) .
$$

Setting $\omega=\exp \{2 \pi i / 3\}$, define

$$
f_{0}\left(a^{n} b^{k}\right)=\left\{\begin{array}{cl}
\frac{1}{3} \omega^{n} & \text { if } k=0, \\
0 & \text { if } k \neq 0,
\end{array}\right.
$$

and

$$
f_{j}(x)=f_{0}\left(x b^{-j}\right) \quad(x \in G ; j=0, \pm 1, \pm 2, \cdots) .
$$

I claim that

$$
f_{0} * f_{j}=f_{j} \text { and } f_{2 m-1} * f_{j}=0
$$

for all integers $j$ and $m$. Indeed,

$$
\begin{aligned}
\left(f_{0} * f_{j}\right)\left(a^{n} b^{j}\right) & =\sum_{r=0}^{2} f_{0}\left(a^{n-r}\right) f_{j}\left(a^{r} b^{j}\right) \\
& =\frac{1}{9} \sum_{r=0}^{2} \omega^{n-r} \cdot \omega^{r}=f_{j}\left(a^{n} b^{j}\right),
\end{aligned}
$$

whereas (5) shows that

$$
\begin{aligned}
\left(f_{2 m-1} * f_{j}\right)\left(a^{n} b^{2 m-1+j}\right) & =\sum_{r=0}^{2} f_{2 m-1}\left(a^{n-r} b^{2 m-1}\right) f_{j}\left(a^{-r} b^{j}\right) \\
& =\frac{1}{9} \sum_{r=0}^{2} \omega^{n-r} \omega^{-r}=0 .
\end{aligned}
$$

If now $c_{m}$ are complex numbers such that $\sum_{-\infty}^{\infty}\left|c_{m}\right|<\infty$, and if

$$
f=f_{0}+\sum_{-\infty}^{\infty} c_{m} f_{2 m-1}
$$

then 


$$
\|f\|=1+\sum_{-\infty}^{\infty}\left|c_{m}\right|<\infty,
$$

and the equations (9) show that $f * f=f$.

Taking infinitely many $c_{m} \neq 0$, we thus obtain idempotents on $G$ with infinite support (and, a fortiori, with infinite support group). The example $f=f_{0}+f_{1}$ shows that there exist idempotents on $G$ with finite support but infinite support group. Equation (11) shows that every number $\geqq 1$ is the norm of some idempotent on $G$. Unless all $c_{m}$ are 0 , the idempotents (10) are not self-adjoint.

III. I have not succeeded in proving the existence of self-adjoint idempotents with infinite support, but it is easy to give examples in which the support group is infinite.

Put

$$
\begin{aligned}
& a=(\alpha \beta \gamma)(12)(34)(56) \cdots, \\
& b=(\alpha \beta \gamma)(23)(45)(67) \cdots .
\end{aligned}
$$

Then $a b$ has infinite order, so that the group $G$ generated by $a$ and $b$ is infinite. The relations $a^{2}=b^{2}, a^{6}=b^{6}=1$ hold. Define $g_{1}\left(a^{n}\right)=\frac{1}{6}$, $g_{1}=0$ elsewhere; $g_{2}\left(b^{n}\right)=\frac{1}{6} \exp \{n \pi i / 3\}, g_{2}=0$ elsewhere. Then

$$
g_{1} * g_{1}=g_{1}, \quad g_{2} * g_{2}=g_{2}, \quad g_{1} * g_{2}=g_{2} * g_{1}=0 .
$$

Hence $g=g_{1}+g_{2}$ is an idempotent on $G$ whose support $S$ is finite. Since $a \in S$ and $b \in S, G$ is the support group of $g$; and since $g_{1}$ and $g_{2}$ are self-adjoint, so is $g$.

IV. Even on a finite group there can be uncountably many idempotents, both self-adjoint and non-self-adjoint. To see this, let $G$ be the noncyclic group of order 6 , with generators $a$ and $b$. The relations $a^{3}=b^{2}=1, b a=a^{2} b$ hold. If $p, q, r$ are complex numbers, subject to

$$
p^{2}+p q+q^{2}=\frac{1}{12}-r^{2}
$$

and if

$$
\begin{aligned}
& f(1)=\frac{1}{3}, \quad f(a)=-\frac{1}{6}+i r, \quad f\left(a^{2}\right)=-\frac{1}{6}-i r, \\
& f(b)=p+q, \quad f(a b)=-p, \quad f\left(a^{2} b\right)=-q,
\end{aligned}
$$

explicit computation shows that $f * f=f$. If $r$ is real and $12 r^{2}<1$, then $p$ and $q$ can be taken real in (14), and the resulting idempotents $f$ are self-adjoint. If $r$ is not real, $f$ is not self-adjoint.

V. We conclude with a positive result: 
THEOREM. If $f$ is an idempotent on $G$ and if $\|f\|=1$, then the support of $f$ is a finite subgroup $H$ of $G$, and

$$
f(x y)=|H| f(x) f(y) \quad(x, y \in H) .
$$

Here $|H|$ denotes the number of elements of $H$. We sketch the proof. Let $S$ be the support of $f$, let $m=\max |f(x)|(x \in G)$, and let $H$ be the set of all $x \in G$ at which $|f(x)|=m$. Clearly $H$ is finite. For $x \in H$, we have

$$
\left|\sum_{y} f(y) f\left(y^{-1} x\right)\right|=m .
$$

Since $\|f\|=1,(17)$ is only possible if $y^{-1} x \in H$ for every $y \in S$, i.e., if $S^{-1} H \subset H$. Since $H \subset S$, it follows that $H$ is a group, and then that $S=H$. Also, $|f(x)|=|H|^{-1}$ on $H$. The equation $f(x)=\sum f(y) f\left(y^{-1} x\right)$ then forces the arguments of $f(y) f\left(y^{-1} x\right)$ to be equal to the argument of $f(x)$, for all $x, y \in H$, and this gives (16).

Since non-negative idempotents have norm 1 or 0 , the above theorem characterizes them as well.

Finally, observe that (16) implies that $f(x y)=f(y x)$ for all $x, y \in G$. In other words, all idempotents of norm 1 lie in the center of the group algebra. It would be interesting to know whether statement (A) of the Introduction is true for all central idempotents.

\section{REFERENCES}

1. P. J. Cohen, On a conjecture of Littlewood and idempotent measures, Amer. J. Math. 82 (1960), 191-212.

2. Walter Rudin, Idempotent measures on abelian groups, Pacific J. Math. 9 (1959), 195-209.

3. - Fourier analysis on groups, Interscience, New York, 1962.

\section{UNIVERSITY OF WISCONSIN}

